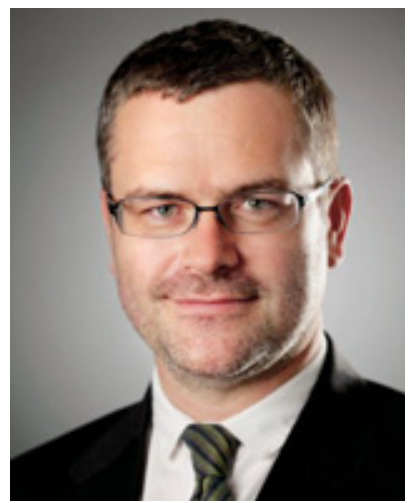

Herr Dr. Schwabl, wo steht die Tibetische Medizin in Asien heute?

Die Situation der Tibetischen Medizin in Asien ist sehr kompliziert. Zum einen ist ja Tibet seit der Annexion durch China 1950 kein unabhängiger Staat mehr. Etwa 6 Millionen Tibeter leben auf einem Gebiet, das etwa 30mal so gross ist wie die Schweiz. Die beiden benachbarten Kulturen China und Indien haben pro Land mehr als 1 Milliarde Einwohner. Die Voraussetzungen für das eigenständige Überleben der tibetischen Kultur, wozu auch die Tibetische Medizin zählt, sind in Anbetracht dieser Kräfteverhältnisse sehr schwierig. Dennoch gibt es eine Reihe von Bemühungen. So wurde 1960 am Sitz der tibetischen Exilregierung in Dharamsala, Indien, das Tibetische Medizininstitut MenTsee-Khang gegründet. Von dort gehen auch nach wie vor die wichtigsten Impulse für die Tibetische Medizin aus.

Wie schätzen Sie die kürzliche Meldung über die offizielle Anerkennung der Tibetischen Medizin durch den indischen Staat ein?

Letztes Jahr wurde die indische Universität Sarnath mit ihrem Lehrgang für Tibetische Medizin akkreditiert.

\title{
Tibetische Medizin in Europa und Asien - Herausforderungen und Chancen
}

\author{
Interview mit Herbert Schwabl, VR-Präsident der PADMA AG
}

Dr. techn. Herbert Schwabl ist Biophysiker und VR-Präsident der PADMA AG in Schwerzenbach. Das Unternehmen, das tibetische Arzneimittel herstellt und vertreibt, konnte im Jahr 2009 sein inzwischen 40-jähriges Bestehen feiern.

Auch in den tibetischen Provinzen Chinas arbeiten Universitäten und Kliniken mit Tibetischer Medizin. In diesem Kontext muss man die offizielle Anerkennung der Tibetischen Medizin durch die beiden asiatischen Grossmächte einordnen. Für die Tibetische Medizin ist es ein wichtiger Schritt in einer politisch heiklen Zeit. Auf jeden Fall ist es nun möglich, dort universitäre Ausbildungs- und Forschungsprogramme zu starten. Erste Schritte wurden eben in Indien und an einigen Universitäten in China (z.B. Lhasa und Xining) unternommen.

Inwiefern sind diese Entwicklungen stellvertretend für die heutige Verbreitung, Anerkennung und Erhaltung der Tibetischen Medizin im Westen?

Die Tibetische Medizin in Europa hat keine Lobby - im Gegensatz z.B. zur TCM. Diese konnte dank uneingeschränkter personeller Ressourcen, ich verweise nochmals auf das unterschiedliche Kräfteverhältnis, hier im Westen mit und auch dank chinesischen Ärzten aufgebaut werden. Es gibt zahlreiche Ausbildungsmöglichkeiten im Westen. Sie hat sich hier etabliert. Wohlgemerkt: Ich stelle diesen Sachverhalt lediglich fest; es ist nicht meine Absicht, diejenigen Kollegen, die chinesische oder indische
Medizin praktizieren oder studieren, zu kritisieren.

Wir arbeiten nun schon mehr als 40 Jahre für die Anerkennung der Tibetischen Medizin. Es ist uns immerhin gelungen, sie im Bewusstsein der Bevölkerung zu verankern. Die Ausbildung zum Tibetischen Arzt/ Therapeuten steckt aber in Europa noch immer in den Kinderschuhen. Hier sind noch grössere Anstrengungen nötig.

Könnten Sie sich Studienprogramme inkl. eines mehrmonatigen Aufenthalts in Tibet - auch in der Schweiz vorstellen bzw. gibt es diese bereits?

Zuerst möchte ich auf die bereits bestehenden Lehrgänge hinweisen: In der Schweiz bietet die InteressensGemeinschaft für Tibetische Medizin ein Ausbildungsprogramm in tibetischer Massage an (www.ig-tibetischemedizin.ch). In Deutschland gibt es seit mehr als 10 Jahren im Rahmen der DÄGfA einen Grundausbildungskurs für Ärzte (www.daegfa.de). Im österreichischen Hüttenberg soll in naher Zukunft ein Ausbildungszentrum für Tibetische Medizin entstehen. Damit sind erste Anfänge gesetzt.

Studienprogramme in Asien sind für Europäer immer auch eine transkulturelle und oft auch anstrengende

\section{KARGER}

Fax +497614520714 
Erfahrung. Für die Tibetische Medizin gibt es dort meines Wissens noch keine modularen Lehrgänge, die auf einem europäischen Studienabschluss aufbauen. Diese zu entwickeln, wird sicher noch einige Zeit dauern.

Wie könnte die Rolle der PADMA AG bei der Etablierung solcher Programme aussehen?

Es ist der PADMA AG ein Anliegen, tibetische Arzneimittel nach internationalen Qualitätsnormen herzustellen und damit westlichen Ärzten und Patienten zugänglich zu machen. Durch unser Engagement auf dem Gebiet der Forschung versuchen wir auch stets, etwas an die tibetische Gesellschaft zurückzugeben: nämlich die internationale Anerkennung auf dem Gebiet der Wissenschaft.

Ausbildungsprogramme werden von Berufsorganisationen und Lehranstalten bereitgestellt. Wir haben nach Möglichkeit stets versucht, Bestrebungen von solchen Organisationen oder Privatpersonen mitzutragen. Ich muss aber festhalten: Unser Kerngeschäft ist die Produktion tibetischer Arzneimittel; hiermit sind wir eigentlich voll ausgelastet. Einerseits sind wir unserem Qualitätsanspruch verpflichtet und andererseits den stetig wachsenden Anforderungen der $\mathrm{Zu}$ lassungsbehörden.

Letztes Jahr wurde die PADMA AG 40 Jahre alt. Wie beurteilen Sie rückblickend die Entwicklungen und die Leistungen der PADMA AG im Bereich der Tibetischen Medizin während dieser Zeit?

In Anbetracht der beiden Systeme Schulmedizin und Tibetische Medizin -, die unterschiedlicher nicht sein könnten, ist es schon erstaunlich, dass wir dieses Jubiläum feiern konnten. Auch wenn wir in den ersten 20 Jahren bis etwa in die 1990er Jahre viel Zeit mit Gerichtsstreitigkeiten verloren haben - es ging um die Kassenzulässigkeit von PADMA 28 -, sind wir jetzt umso engagierter, neue Rezepturen zur Zulassung zu bringen. In den vergangenen Jahren konnte gezeigt werden, dass sich die Tibetische Medizin unter kontrollierten Bedingungen, wie sie in einer Studie gefordert sind, untersuchen lässt. Das gibt uns die Hoffnung, mit dem Erfahrungsschatz der Tibetischen Medizin neue Wege in der Therapie zu erschliessen.

Im Jahr 2005 war Seine Heiligkeit der 14. Dalai Lama bei Ihnen und der PADMA AG zu Besuch: Wie schätzen Sie die Signalwirkung dieses Besuches für PADMA und die Tibetische Medizin im Westen ein?

Die Signalwirkung war sicherlich im Westen genauso gross wie im Osten. Seine Heiligkeit hat sich bei seinem Besuch für unsere wissenschaftliche Tätigkeit interessiert. Er hat uns auch ermuntert, weiter damit fortzufahren. Für ihn ist die Tibetische Medizin ein Weltkulturerbe, welches allen Menschen auf dieser Erde offenstehen sollte. Weiters betonte er, dass tibetische Arzneien aufgrund ihrer speziellen Zusammensetzung, etwa Heilkräuter und Mineralien, auf materieller Ebene wirksam sind.

Damit öffnete er Türen für die moderne Wissenschaft, diese Rezepturen zu untersuchen. Deren Methoden müssen sicherlich nicht nur reduktionistisch angewendet werden. Aber auch wir sind davon überzeugt, dass sich die Wirkprinzipien tibetischer Arzneien wissenschaftlich fassen und erklären lassen.

Welche Schritte wurden bereits bezüglich einer europaweiten Vernetzung im Bereich der Tibetischen Medizin unternommen? Wie nötig ist eine Vernetzung Ihrer Meinung nach?
Ich kann behaupten, dass wir nahezu mit allen Personen, die sich in Europa mit Tibetischer Medizin befassen, in Kontakt stehen. Als kleine Gruppe sind wir gut vernetzt. Auf europäischer Ebene verlagern sich jedoch immer mehr Entscheidungen nach Brüssel. Hier müssen wir mit der gesamten komplementärmedizinischen Bewegung erkennen, dass nur ein gemeinsames Auftreten vor dem Europäischen Parlament, vor der Europäischen Kommission, Wirkung zeigt. Wir müssen uns also in diesen gesamteuropäischen Prozess einordnen, um für die Komplementärmedizin Freiräume zu erkämpfen (Patientenrechte, Ausbildung von Ärzten, Herstellung von Arzneimitteln). Sich als Kleinunternehmen auf dem EU-Parkett zu bewegen - und noch dazu aus der Schweiz -, ist kein leichtes Unterfangen.

Wie schätzen Sie die momentane Rolle der Tibetischen Medizin und deren Wahrnehmung in der schweizerischen Bevölkerung ein?

Unsere Produkte PADMA 28 und PADMA LAX sind einem Grossteil der Schweizerinnen und Schweizer über 40 Jahre bekannt. Viele wissen anfangs oft gar nicht, dass das von ihrem Arzt verschriebene Arzneimittel PADMED CIRCOSAN oder PADMED LAXAN aus der Tibetischen Medizin stammt. Wer vom tibetischen Ursprung weiss, steht unseren Produkten meist mit einem grossen Wohlwollen gegenüber. Am Rand mag das sicherlich mit der humanitären Tradition der Schweiz zusammenhängen. In den 70er Jahren wurden hier viele Flüchtlinge aufgenommen und nach wie vor lebt die grösste Tibetergemeinschaft in Europa in der Schweiz und hat sich vorbildlich integriert. Für mich ist aber nach wie vor die Wirkkraft der tibetischen Arzneien das wichtigste Argument. 
Wie sieht die Zukunft der Tibetischen Medizin im Westen Ihrer Meinung nach aus? Welche Rolle wird die PADMA AG dabei spielen?

Wie es mit der Tibetischen Medizin im Westen zukünftig weitergeht, hängt im Moment von einigen wichtigen politischen und behördlichen Entscheiden ab. In der Schweiz geht es nach der gewonnenen Abstimmung «Ja zur Komplementärmedizin» um die Umsetzung der Forderungen: erleichterte Zulassung von Heilmitteln der Komplementärmedizin, Ausbildungsreglemente für Komplementärmedizin. Wir werden versuchen, möglichst vorausschauend zu agieren, um den Bestand der tibetischen Arzneimittel in der Schweiz mindestens über die nächsten 40 Jahre zu sichern. Die politische Arbeit ist also wichtiger geworden. Aus diesem Grund engagiere ich mich auch persönlich im neuen «Dachverband Komplementärmedi- zin». Was sich derzeit politisch in der kleinen Schweiz in Sachen Komplementärmedizin tut, ist einzigartig. Hier kann Europa noch viel lernen, aber es liegt an uns, die gewonnenen Erkenntnisse auch über die Grenzen nach aussen zu tragen.

Welche Bedeutung hat die Tibetische Medizin für Sie persönlich?

Als ich zum ersten Mal an der Universität von der Tibetischen Medizin gehört habe, war das für mich ein spannendes Gebiet. Je mehr ich mich damit beschäftigte, desto mehr kam ich auch unweigerlich mit Patienten und ihren Geschichten in Kontakt. Ich hörte von Menschen, die schlimme Erkrankungen wie Herzinfarkte hatten oder kurz vor einer Bypassoperation standen. Der Arzt hat ihnen PADMED CIRCOSAN verschrieben - oder vom Bekanntenkreis wurde PADMA 28 empfohlen. Das klingt für Aussenstehende manchmal unglaublich, aber diese Menschen haben uns berichtet, wie sich ihr Zustand enorm gebessert habe. Wenn sich solche Geschichten häufen, auch Ärzte davon berichten und in der Folge Forscher neue Ergebnisse dazu beitragen, dann beginnt man zu erkennen, dass mehr dahinter steckt. Altes Erfahrungswissen ist das kulturelle Erbe der Menschheit. Wir leben in der Moderne, aber für mich gehört altes Wissen zum Werkzeugkasten genauso dazu, denn es gibt immer wieder neue Herausforderungen. Für mich wurde die Beschäftigung mit der Tibetischen Medizin zur Faszination.

Herr Dr. Schwabl, vielen Dank für das Interview!

Interview: Alexander Eitner 